\title{
El currículum y la innovación educativa: primeras notas sobre la Nueva Escuela Mexicana
}

Rubén Madrigal Segura

ruben.madrigal@isceem.edu.mx

Instituto Superior de Ciencias de la Educación del Estado de México-Sede Toluca

Recibido: 17 de abril del 2020

Aceptado: 30 de abril 2020

\section{Resumen}

Este ensayo es producto de la reflexión generada para una conferencia en un Seminario de currículum e innovación educativa. La nueva Escuela Mexicana es la denominación recibida de la actual reforma educativa de la cual solamente se cuenta con el marco normativo de ella. Conocer el marco normativo de la reforma nos da pista de la manera en que los diseñadores del currículum lo van a plasmar en los planes y programas de estudio. Lo anterior lleva a plantearnos ¿a qué época histórica representa la Nueva Escuela Mexicana? ¿cuál es el modelo de ciudadano formado con el currículum actual? La utilidad de la metáfora de Herder sobre la semilla y la planta no sirve para pensar en el currículum, la innovación educativa y la Nueva Escuela Mexicana como un árbol que habría que analizar sobre los elementos que lo constituyen para entenderlos. La innovación educativa concebida actualmente requiere innovarse en el actual cambio de época histórica; el currículum debe de pensarse desde los sujetos del desarrollo curricular, es decir de aquellos profesores que se constituyen como sujetos sociales, sin menospreciar lo que los sujetos de determinación y diseño curricular aportan; por ende, la Nueva Escuela Mexicana requiere de un nuevo profesor que se adhiera a un proyecto educativo por escuela o zona escolar, a una comunidad es decir a otros profesores con ideas parecidas y a una utopía que la educación sigue siendo un factor de felicidad, por lo menos.

Palabras clave: currículum, innovación educativa y reforma educativa. 


\title{
Curriculum and educational innovation: first notes on the New Mexican School
}

\begin{abstract}
This essay is the product of the reflection generated for a conference in a seminar on curriculum and educational innovation. The new Mexican School is the name received from the current educational reform of which only the normative framework of it is available. Knowing the regulatory framework of the reform gives us clue to how the designers of the curriculum will translate it into the curriculum and programs. This leads us to consider what historical era does the New Mexican School represent? What is the model of citizen trained with the current curriculum? The usefulness of Herder's metaphor on seed and plant does not serve to think about the curriculum, educational innovation and the New Mexican School as a tree that would have to be analyzed on the elements that constitute it to understand them. The educational innovation conceived today requires innovating in the current change of historical era; the curriculum must be thought of from the subjects of curriculum development, that is, those teachers who constitute themselves as social subjects, without undermining what the subjects of determination and curriculum design provide; therefore, the New Mexican School requires a new teacher who adheres to an educational project by school or school zone, to a community i.e. other teachers with similar ideas and a utopia that education remains a factor of happiness, at least.
\end{abstract}

Keywords: curriculum, educational innovation and educational reform.

\section{Introducción}

El presente ensayo tiene su antecedente en una conferencia a la cual fui invitado por la colega María del Rosario Castañeda Reyes para impartirla en el marco del Seminario Currículum e Innovación Educativa. A partir de esa conferencia realizada en el mes de diciembre de 2019, ha publicado la Secretaría de Educación Pública el Programa Sectorial de Educación (PSE) 2019-2024, el cual deriva del Plan Nacional de Desarrollo (PND) 2019-2024 aprobado por el decreto publicado en el Diario Oficial de la Federación (DOF) el 12 de julio de 2019. 
Lo anterior es relevante en cuanto va a normar lo que se va a plasmar en los planes y programas de estudio de los diferentes niveles educativos en su carácter de educación obligatoria. En ese sentido, nos atrevemos a pensar un antes y un después en torno a lo educativo y la educación. Siguiendo a Carbajal (2003) lo educativo lo podemos encontrar en las estructuras sociales, lo educativo es necesario en la estructura, sin embargo, se geo localiza en la escuela con su currículum. El interés del escrito va más allá de un partido en el poder político, sino en los planteamientos en torno al currículum, la innovación educativa y la Nueva Escuela Mexicana (NEM) que se delinea en el marco normativo que va a impactar en el Sistema Educativo Nacional.

El nuevo gobierno de nuestro país como cada sexenio plantea su propia postura en torno a la educación a lo cual nombran reforma educativa, en este caso le denominan la Nueva Escuela Mexicana ¿A qué época histórica representa la Nueva Escuela Mexicana? ¿Cuál es el modelo de ciudadano formado con el currículum e innovación educativa con el modelo actual?

El currículum es un concepto que se acomoda a las necesidades de las políticas educativas de los gobiernos y de los organismos internacionales (OCDE, FMI y BM, por mencionar algunos), además de las posturas de las tribus académicas. Partimos de manera inicial de la definición que De Alba (2002) hace del currículum:

Entendemos a la síntesis de elementos culturales (conocimientos, valores, costumbres, creencias, hábitos) que conforman una propuesta político-educativa pensada e impulsada por diversos grupos y sectores sociales cuyos intereses son diversos y contradictorios, en donde algunos de estos son dominantes y otros tienden a oponerse y resistirse a tal dominación o hegemonía. (p. 52).

En ese sentido, en el diseño y en la determinación de un currículum intervienen: Mexicanos Primero, Coparmex, la Unión Nacional de Padres de Familia, Provida, la iglesia católica, la SEP y el Sindicato Nacional de Trabajadores de la Educación, entre otras organizaciones. 
Siguiendo a Herder (2007): "la semilla cae en la tierra y desparece; el embrión se forma en lo oculto, como tal vez no lo aprobaría a priori la lente del filósofo, y la planta surge totalmente formada" (p. 26). Lo anterior nos lleva a pensar el currículum, la innovación y la reforma educativa como si fueran un gran árbol que requiere rastrear sus raíces y su semilla. Luego entonces ¿qué vale la pena pensar del currículum, de la innovación y de la Nueva Escuela Mexicana?

La estructura del escrito se encuentra articulado en cuatro apartados. El primero donde se abre el debate de la innovación educativa creada en una época histórica y que ya no responda a los nuevos tiempos. El segundo apartado se discute de manera rápida el concepto de currículum que desarrollan los profesores como sujetos. El tercer apartado con los elementos normativos que dejan visibilizar elementos de la Nueva Escuela Mexicana. En el cuarto apartado de plantea una interrogante para continuar la discusión que otros han abierto y que ahora la desplazamos hacia la Nueva Escuela Mexicana. Por último, se presentan a manera de cierre y apertura unas conclusiones temporales.

\section{La innovación educativa}

La pregunta clásica ¿qué es la innovación? Innovar es un cambio que introduce novedad. Es decir, hacemos referencia a modificar elementos que existe con el fin de mejorar o renovar. Entiendo por innovación educativa a un proceso donde el principal ingrediente es la creatividad y que implica asumir riesgos pues no se conocen los senderos y resultados, lo cual nos pueden llevar a lugares no favorables de acuerdo a lo planeado.

En ese sentido podemos hablar de dos tipos de innovaciones: las curriculares y las generadas en el aula que, podemos decir atiende a lo pedagógico-didáctico. En relación a la innovación curricular Frida Díaz Barriga y Arceo dice:

Para entender el sentido de la innovación en la lógica de los modelos curriculares, hay que precisar que un modelo de educativo es una construcción teórica, un prototipo y una representación idealizada de un proceso que describe su funcionamiento y permite la prescripción de un cauce de acción. (p. 119). 
Así podemos distinguir el currículum por competencias, flexibilidad curricular, currículo centrado en el aprendizaje de los alumnos, transversalidad del curricular, entre otros.

Por otro lado, las innovaciones generadas en el aula se pueden distinguir dos: las innovaciones generadas por el mercado; y las generadas desde las iniciativas de los propios profesores. Las innovaciones han sido construidas con la "idea de desarrollo" de la calidad de la educación. Lo que ha llevado a cambiar cada sexenio la ruta de la educación a través de ajustes o cambios curriculares: nuevos procesos de enseñanza, nuevos contenidos de aprendizaje, además de prácticas y organizaciones de trabajo.

Siguiendo a De Souza (2005) para comprender la crisis actual del currículum y la innovación, debemos atrevernos a pensar que son la herencia del colonialismo europeo y de la modernidad. Por un lado, el colonialismo ha justificado la dominación física, cultural e intelectual de unos pueblos sobre otros con el pretexto de la raza: unos blancos-cristianos superiores y otros indios o de otro color como inferiores. La modernidad se creó con la idea de universalismo como una forma de ser, sentir, pensar y actuar desde Europa. Esas dos ideas las hemos incorporado a un imprinting cultural como un modelo de desarrollo a través de la educación, la ciencia y la tecnología en nuestro país. El universalismo desplaza la idea de elaborar un currículum por región a pesar de que nuestro país es un enorme mosaico cultural, físico y poblacional.

Vamos a denominar "modo clásico" a la manera de hacer un currículum para todo el país y a la innovación concebida en otro momento histórico y que va perdiendo su monopolio, no digo que ha desaparecido. Ese "modo clásico" generado en Europa es un obstáculo para generar la mejora en las escuelas de nuestro país, porque lo que promueve es un constante cambio surgido como parte del problema-solución.

\section{El currículum obligatorio}

La idea de currículum se ha extendido en los círculos académicos a partir de los años cincuenta del siglo pasado. El gobierno federal se ha caracterizado por realizar cambios en materia curricular cada sexenio. Lo que lleva a constantes cursos de actualización para que 
los profesores encargados de su desarrollo se encuentren actualizados con las modificaciones realizadas. Se cambia el currículum pretendiendo con ello hacer que cambien las circunstancias relacionadas con la educación y lo educativo: la violencia en todas sus manifestaciones, los valores que debemos poseen como miembros de una sociedad, la contaminación en todas sus modalidades, entre otras problemáticas sociales.

Los contenidos plasmados en los planes y programas de estudios son importantes ya que es una manera de mirar lo que el gobierno considera valioso para la educación escolarizada. Lo anterior es parte de los elementos culturales que plantea De Alba (2002) en su definición de currículum. Otro elemento son los valores, en el caso de la NEM plantea el Programa Sectorial de Educación 2019-2024 que la regeneración moral de nuestro país sólo es posible "a partir de una orientación integral basada en valores como la honestidad, honradez, ética, libertad y confianza, principios rectores del PND 2019-2024 (p. 8). Es decir, teniendo presente en la escuela que el combate a la corrupción somos sujetos implicados en el problema y solución de la corrupción en todos sus niveles y modalidades.

Las costumbres se arraigan en la población como otro de los elementos culturales que vale la pena visibilizar en el profesorado. La documentación de dos experiencias realizadas por Espinosa (2011) sobre la actualización de Formación Cívica y ética en 1999 y la de 2005 en educación secundaria en Jalisco que se realizó en cascada. La capacitación en cascada se ha vuelto una costumbre en el Sistema Educativo Nacional. Por otro lado, esa capacitación se genera con "Los equipos encargados de organizar la estrategia de capacitación se integran a partir de ciertos perfiles profesionales del personal técnico-pedagógico, por designación de la autoridad en turno, y se desintegran una vez concluida la tarea (Espinoza, 2011, p. 1). La falta de personal ex professo para la realización de la capacitación de las nuevas reformas es un pendiente en todos los niveles educativos.

El último elemento cultural son los hábitos. La práctica muy común entre el profesorado se deja ver en los cursos de actualización que esperan que el conductor les diga el "como" resolver sus problemas de enseñanza aprendizaje generados a partir de los 
requerimientos de la reforma educativa en turno. Se asumen como profesores pasivos que esperan que alguien les resuelva sus incognitas.

\section{La Nueva Escuela Mexicana}

¿Podemos visibilizar detalles de un cambio de época histórica? La reforma al artículo $3^{\circ}$ constitucional marca un antes y un después en su visión utópica. Del artículo mencionado rescato cuatro ideas, la primera dice: "la educación se basará en el respeto irrestricto de la dignidad de las personas, con un enfoque de derechos humanos y de igualdad sustantiva" (SEP, 2019, p. 24). Desde donde lo vemos, se marca un fin relacionado con la convivencia humana. Este humanismo que nos marca no puede ser el que planteaba Hidegger en Alemania en 1946; en el mismo sentido la crisis del humanismo plasmada por Samuel Ramos entre lo material y lo espiritual también se encuentra distante del acontecer actual. No se trata de resolver un problema de la modernidad desde dentro de ella, sino de visibilizar un cambio de época que se encuentra en una etapa embrionaria.

Las relaciones humanas se generan a través del amor, dice Maturana. De ser así, tenemos que visibilizar los diferentes tipos de amor: filial, carnal, por el otro, pedagógico, por el estudio, por los animales y la naturaleza. La crisis actual de la humanidad tiene que pensarse en términos de ¿qué vale la pena pensarse sobre el nuevo humanismo? El nuevo humanismo tiene que plantearse las formas pertinentes para resolver problemas planetarios que atentan contra la propia humanidad, los problemas locales deben de atender los problemas globales de subsistencia de la especie humana.

En la segunda idea se asegura que "El Estado priorizará el interés superior de niñas, niños, adolescentes y jóvenes en el acceso, permanencia y participación en los servicios educativos" (SEP, 2019, p. 24), entiendo que el Estado se convierte en un estado educador, no sólo para la educación básica, sino para todos los niveles educativos. Lo anterior lleva 
consigo retos, tensiones y aporías. Por otro lado, se continúa pensando en un currículum centrado en el alumno, para verlo concretado en planes y programas de estudio hay que esperar a que se materialicen.

La ley que reglamenta el artículo tercero en materia de Mejora Continua de la Educación plasma que uno de los principios del Sistema es:

El aprendizaje de las niñas, niños, adolescentes y jóvenes, como centro de la acción del Estado para lograr el desarrollo armónico de todas sus capacidades orientadas a fortalecer su identidad como mexicanas y mexicanos, responsables con sus semejantes y comprometidos con la transformación de la sociedad de que forman parte. (p. 2).

Habrá que esperar la profundidad y la manera en que los desarrolladores curriculares logren plasmar que los alumnos son el centro de la actividad escolar. Por otro lado, a nuestro juicio lo más importante de lo anterior es el compromiso que asuman los profesores ya que son el alma de la NEM y tienen que prepararse para asumir el rol protagónico, de ser así, siguiendo a De Alba (2002), en su papel de sujetos del desarrollo curricular, capaces de darle vida a lo que surja en los planes y programas de estudio.

Tercera idea digna de analizarse del artículo $3^{\circ}$ constitucional dice: "las maestras y los maestros son agentes fundamentales del proceso educativo y, por tanto, se reconoce su contribución a la trasformación social” (SEP, 2019, p. 24). Esta misma idea la plasman en el tercer principio en la Ley de Mejora Continua de la Educación y en el tercer objetivo prioritario del Plan Sectorial de Educación 2019-2024. Es decir, se reconoce que no puede existir un acuerdo para la transformación social que pretende el actual gobierno, sin la participación consciente del profesorado como un sujeto del desarrollo curricular. Muchos de esos profesores nos podemos encontrar en proceso de constitución como sujeto de desarrollo curricular para la transformación social. Sin olvidar que puede haber resistencias, tensiones, retrocesos y avances. 
La cuarta idea vertida en el artículo tercero que deseo destacar es "Los planes y programas de estudio tendrán perspectiva de género y una orientación integral, por lo que se incluirá el conocimiento de las ciencias y humanidades" (SEP, 2019, p. 25). Por primera vez se materializa una demanda de un sector de la sociedad mexicana relacionada con la perspectiva de género y una orientación integral relacionada con las humanidades, principalmente la filosofía, la literacidad, la innovación y las lenguas, tanto extranjera como indígenas de nuestro país.

En el sentido anterior el artículo 16 de la Ley de Mejora Continua de la Educación, habla de la oferta de formación, capacitación y actualización deberá: en el apartado VIII incorporar la perspectiva de género y el enfoque de derechos humanos. Lo que permite esperar que los profesores van a tener la oportunidad de actualizarse, capacitarse y formarse en esos rubros.

\section{¿Quo vadis el currículum y la innovación de la innovación en educación?}

Comparto la idea de Alicia de Alba de que los profesores tenemos la posibilidad de convertirnos en sujetos del desarrollo curricular. Puntualizo, no todos los profesores somos sujetos porque para ello tenemos que reconocer que un sujeto se constituye como tal al participar en una comunidad con un proyecto y de una utopía. En ese sentido un sujeto del desarrollo curricular que deviene en profesor debe ser parte de un proyecto educativo. Segundo, ese proyecto es parte de una utopía que nos permita generar acciones. Tercero, el proyecto y la utopía nos hace ser parte de una comunidad, es decir, nos une a varios sujetos en algo que nos es común, la educación y lo educativo.

Si bien no somos sujetos de determinación curricular y muchos menos de construcción formal del currículum, podemos incidir en una gestión curricular crítica que permita desarrollar un currículum que rescate ideas pensadas sobre la educación que rompa con la pasividad de la comunidad. La gestión curricular consiste en la implementación de proyectos de formación continua que permita a los profesores constituirse como sujetos: con proyectos, utopías, finalidades y retos; que les permitan valorar a la filosofía de la educación, 
los planes y programas de estudio, las leyes secundarias y los reglamentos relacionados con la educación para que, a partir de una visión amplia se pueda incidir en la práctica curricular desde una visión práctica-teoría-práctica con una posición de cultura reflexiva y de concienciación.

\section{A manera de cierre y apertura}

Pensar que el currículum es todo aquellos que realizar los diseñadores y que toman en cuenta las ideas de quienes determinan lo que debe integrar el currículum es dejar fuera a los sujetos que con su experiencia van a desarrollarlo de tal forma que se convierten en el centro del mismo. Lo anterior se fundamenta en la convicción de la mejor opción en los tiempos en que cada sexenio se modifica el currículum de la educación obligatoria.

Por lo anterior, se requiere un tipo de profesor que sea capaz de convertirse en sujeto del desarrollo curricular versus trabajador de la educación o cualquier otro nombre que se le quiera dar: mediador, profesional de la educación, agente de cambio, etcétera. Ese sujeto del desarrollo curricular entenderá que la innovación de la educción también requiere de innovarse porque no responde a las exigencias de la época.

En esa lógica lo que pretendemos potenciar con la idea de sujetos del desarrollo curricular ¿educar a la humanidad o humanizar la educación? La respuesta es humanizar a la educación. Porque la educación es realizada por humanos y para humanos, intentando visibilizar que las relaciones humanas se generan en la educación y permean lo educativo. Es más económico formar sujetos del desarrollo curricular que cada sexenio hacer cambios al currículum de la educación obligatoria.

\section{Referencias}

Carbajal, J. (2003). Internet, lo educativo y la educación: complejo discursivo. En Granja, J (Comp.) Miradas a lo educativo: exploraciones en los límites. México. Plaza y Valdés.

De Alba, A (2002). Currículum, crisis, mito y perspectivas. México: CESU-UNAM. 
De Souza, J. (2005). La innovación de la innovación institucional. Ecuador: Red Nuevo Paradigma.

Diario Oficial de la Federación. (2019). Ley reglamentaria del artículo $3^{\circ}$ de la Constitución Política de los Estados Unidos Mexicanos en materia de Mejora Continua.

Espinosa, F. (2011). Organización de los procesos emergentes para la actualización docente en el marco de dos reformas curriculares. COMIE: Nuevo León.

Díaz-Barriga, F. (2013). "Innovaciones curriculares”. En: Ángel Díaz Barriga (Coordinador general). La investigación curricular en México 2002-2011. México. ANUIES. COMIE. Colección Estados del Conocimiento. pp. 109-196

Herder, J. (2007). Filosofía de la historia. Para la educación de la humanidad. España: Ediciones Espuela de la Plata.

Maturana, H. (1997). Emociones y lenguaje en educación y política. Granica: Chile.

Secretaria de Educación Pública. (s/f). Programa Sectorial de Educación 2019-2024. Secretaria de Educación Pública: México.

Secretaría de Educación Pública. (2019). Guía de trabajo para el Taller de capacitación "Hacia una Nueva Escuela Mexicana". Secretaria de Educación Pública: México. 